\title{
Visual Attention Modulates Glutamate-Glutamine Levels in Vestibular Cortex: Evidence from Magnetic Resonance Spectroscopy
}

\author{
${ }^{D}$ Sebastian M. Frank, ${ }^{1,2,3}$ Lisa Forster, ${ }^{1}$ Maja Pawellek, ${ }^{1}$ Wilhelm M. Malloni, ${ }^{1}$ Sinyeob Ahn, ${ }^{4}$ Peter U. Tse, ${ }^{2}$ and \\ ${ }^{-1}$ Mark W. Greenlee ${ }^{1}$ \\ ${ }^{1}$ Institute for Experimental Psychology, University of Regensburg, 93053 Regensburg, Germany, ${ }^{2}$ Department of Psychological and Brain Sciences, \\ Dartmouth College, Hanover, New Hampshire $03755,{ }^{3}$ Department of Cognitive, Linguistic, and Psychological Sciences, Brown University, \\ Providence, Rhode Island 02912, and ${ }^{4}$ Siemens Healthcare, San Francisco, California 94123
}

Attending to a stimulus enhances the neuronal responses to it, while responses to nonattended stimuli are not enhanced and may even be suppressed. Although the neural mechanisms of response enhancement for attended stimuli have been intensely studied, the neural mechanisms underlying attentional suppression remain largely unknown. It is uncertain whether attention acts to suppress the processing in sensory cortical areas that would otherwise process the nonattended stimulus or the subcortical input to these cortical areas. Moreover, the neurochemical mechanisms inducing a reduction or suppression of neuronal responses to nonattended stimuli are as yet unknown. Here, we investigated how attention directed toward visual processing cross-modally acts to suppress vestibular responses in the human brain. By using functional magnetic resonance spectroscopy in a group of female and male subjects, we find that attention to visual motion downregulates in a load-dependent manner the concentration of excitatory neurotransmitter (glutamate and its precursor glutamine, referred to together as Glx) within the parietoinsular vestibular cortex (PIVC), a core cortical area of the vestibular system, while leaving the concentration of inhibitory neurotransmitter (GABA) in PIVC unchanged. This makes PIVC less responsive to excitatory thalamic vestibular input, as corroborated by functional magnetic resonance imaging. Together, our results suggest that attention acts to suppress the processing of nonattended sensory cues cortically by neurochemically rendering the core cortical area of the nonattended sensory modality less responsive to excitatory thalamic input.

Key words: attention; magnetic resonance spectroscopy; parietoinsular vestibular cortex; suppression; thalamus; vestibular system

\section{Significance Statement}

Here, we address a fundamental problem that has eluded attention research for decades, namely, how the brain ignores irrelevant stimuli. To date, three classes of solutions to this problem have been proposed: (1) enhancement of GABAergic interneuron activity in cortex, (2) downregulation of glutamatergic cell activity in cortex; and (3) downregulation of neural activity in thalamic projection areas, which would then provide the cortex with less input. Here, we use magnetic resonance spectroscopy in humans and find support for the second hypothesis, implying that attention to one sensory modality involves the suppression of irrelevant stimuli of another sensory modality by downregulating glutamate in the cortex.

Received Aug. 1, 2020; revised Nov. 12, 2020; accepted Dec. 22, 2020.

Author contributions: S.M.F., P.U.T., and M.W.G. designed research; S.M.F., L.F., M.P., and W.M.M. performed research; W.M.M. and S.A. contributed unpublished reagents/analytic tools; S.M.F. analyzed data; S.M.F., P.U.T., and M.W.G. wrote the paper.

This research was supported by the Deutsche Forschungsgemeinschaft (DFG; Core Facility Grant INST 89/393-1; DFG Grant GR 988/25-1 to M.W.G.) and the National Science Foundation (Grant 1632738 to P.U.T.). P.U.T. and S.M.F. were supported by the Alexander von Humboldt Foundation. M.W.G. was supported by the Harris Distinguished Visiting Professorship Program of Dartmouth College. We thank Christian Renner from the mechanical workshop at the University of Regensburg for construction and maintenance of the CVS device.

S.A. is an employee of Siemens. The authors declare no other competing financial interests. Correspondence should be addressed to Sebastian M. Frank at sebastian_frank@brown.edu. https://doi.org/10.1523/JNEUROSCI.2018-20.2020

Copyright $\odot 2021$ the authors

\section{Introduction}

Attention directed toward a feature or a location enhances the neuronal responses to that feature or location, thus improving the processing of the attended sensory signal (Kastner et al., 1998; Tootell et al., 1998; Carrasco, 2011). However, while enhancing attended signals, attention also acts to suppress unattended sensory cues that might interfere with processing of the attended cue (Frank and Greenlee, 2018). This active ignoring mechanism occurs not only between attended and nonattended features or locations within a sensory modality (e.g., visual signals related to color and motion direction; Saenz et al., 2002), but also cross- 
modally between attended and nonattended signals arising within different sensory systems (e.g., between attended visual and nonattended vestibular signals; Frank et al., 2016a, 2020).

Brandt et al. (1998) observed that the regional cerebral blood flow (rCBF) in parietoinsular vestibular cortex (PIVC), a core cortical area of the vestibular system (Chen et al., 2010; Lopez et al., 2012; Frank and Greenlee, 2018), decreased during sensations of vection induced by optokinetic stimulation compared with conditions of optokinetic stimulation without sensations of vection and fixation without optokinetic stimulation. They referred to this cross-modal decrease of rCBF in PIVC as a deactivation, potentially reflecting an inhibition of PIVC. In our previous studies (Frank et al., 2016a, 2020), we used a visual attentional tracking paradigm in which subjects were asked to attentively track a subset of randomly moving disks as targets among randomly moving distractor disks. We observed a similar crossmodal deactivation of PIVC during visual attentional tracking as shown by a negative functional magnetic resonance imaging (fMRI) blood oxygenation level-dependent (BOLD) signal change during tracking relative to control conditions of passive viewing of randomly moving disks and central fixation without any attended visual motion cues. The designs of our studies and the original study by Brandt et al. (1998) differ in terms of the visual stimulation conditions and the engagement of the subjects with a task (active visual attentional tracking vs passive viewing). Despite these differences, these studies together suggest that visual attentional processing without vestibular motion cues cross-modally deactivates PIVC. Whether this deactivation reflects an inhibition of PIVC remains uncertain. The results of our previous study (Frank et al., 2020) suggest that the deactivation of PIVC during visual attentional tracking is modulated in magnitude by parietal cortex, indicating that the deactivation of PIVC is the result of a topdown suppression. Therefore, we use the term "suppression" in this article to refer to the cross-modal deactivation of PIVC during visual attentional tracking.

It has remained unresolved just how this cross-modal suppression of processing in PIVC is implemented mechanistically. One possibility is that attention might act to suppress processing in PIVC cortically, by increasing the activity of GABAergic inhibitory interneurons (Roux and Buzsáki, 2015) within PIVC, thus rendering the suppressed PIVC less likely to respond to excitatory subcortical input. A second possibility is that attentional suppression might be associated with a downregulation of the activity of glutamatergic cells in PIVC. And a third possibility is that attentional suppression might occur subcortically by inhibiting the ability of thalamic neurons to relay excitatory sensory signals to PIVC.

In this study, we used functional magnetic resonance spectroscopy (fMRS), a recently advanced imaging technology (Duncan et al., 2014; Stanley and Raz, 2018), to investigate the neurochemical mechanisms underlying cross-modal suppression of processing in PIVC in human subjects. fMRS detects changes in the concentrations of chief excitatory (glutamate and its precursor glutamine, referred to together as Glx) and inhibitory (GABA) neurotransmitters (Petroff, 2002; Rothman et al., 2003) in a specific brain area during different experimental conditions, providing insights into the neurochemical mechanisms underlying the sensory and cognitive processes that occur during a task. Here, by using fMRS we investigated the neurochemical mechanisms underlying how attention directed toward visual processing can crossmodally suppress vestibular processing in PIVC.

\section{Materials and Methods}

Subjects. A total of 40 subjects participated in this study (mean \pm SE age, $24 \pm 1$ years; 26 females). For the subset of 20 subjects who completed the caloric vestibular stimulation (CVS) localizer experiment for the definition of PIVC (see below), handedness was determined by the Edinburgh handedness inventory (Oldfield, 1971). The mean right handedness score $( \pm \mathrm{SE})$ was $93.9 \pm 2.72($ minimum $=54$, maximum $=100$ ). All other subjects reported being right handed by self-report. A subset of 16 subjects participated in a previous study (Frank et al., 2020) for which they completed the CVS, temperature, and visual motion localizer experiments. Subjects gave informed written consent before participation. The study was approved by the local ethics boards at the University of Regensburg and Dartmouth College.

Experimental design. Three experiments and several localizer scans were conducted. In a first experiment, a group of 20 subjects ( 12 females) performed visual attentional tracking during fMRI. In a second experiment, a group of 20 different subjects (14 females) performed visual attentional tracking during fMRS. In a third experiment, a subset of 18 subjects from the second experiment performed visual attentional tracking with simultaneous CVS during fMRI. BOLD and fMRS signals were measured in right PIVC. Right PIVC was chosen because previous results suggested that PIVC in the right hemisphere is dominant in right-handed subjects (Dieterich et al., 2003). For subjects in the first experiment, PIVC was defined anatomically using previously published coordinates (Frank et al., 2016b). For subjects in the second and third experiments, PIVC was defined functionally by independent CVS localizer experiments. Additional localizer experiments were conducted in these subjects to confine the definition of PIVC to voxels that are primarily responsive to vestibular rather than temperature or visual motion cues (see below).

Attentional tracking task. Subjects performed a visual attentional tracking task under low- and high-attentional load conditions (Pylyshyn and Storm, 1988; Culham et al., 2001; Frank et al., 2016a; Fig. 1a). This task was chosen, because previous results showed that vestibular sensations of self-motion and area PIVC are strongly suppressed during increasing loads of visual attentional tracking (Frank et al., 2016a, 2020). Furthermore, by using a visual attentional tracking paradigm, the visual attentional load can be varied parametrically while keeping the visual input constant across attentional loads. On each trial of the attentional tracking task, eight white disks (diameter, $0.6^{\circ}$ ) were arranged circularly in the visual periphery for a total of $2 \mathrm{~s}$ (distance from central fixation, $5^{\circ}$ ). During this time, target disks were shown in green while distractor disks were shown in white. One to four disks could be assigned as targets for tracking. The more targets indicated, the greater was the attentional load. Tracking one and two disks served as the low-attentional load condition, while tracking three and four disks served as the high-attentional load condition. After cueing, target disks turned white and were physically indistinguishable from distractors. Then, all eight disks moved randomly (speed, $6 \%$ ) across the screen for a total of $14 \mathrm{~s}$ (screen size for fMRI experiment with attentional tracking, $27^{\circ} \times 20^{\circ}$; screen size for fMRS experiment and fMRI experiment with CVS, $24^{\circ} \times 18^{\circ}$; display size varied because of different scanner setups). Subjects were instructed to covertly track the targets with their attention while maintaining fixation on a fixation spot present at the center of the screen. The moving disks never collided or overlapped and were repelled from central fixation and from the borders of the screen. If subjects inadvertently lost track of a target they were instructed to track another disk to keep the number of tracked disks constant across the trial. At the end of the tracking period, all disks stopped moving and only one disk was highlighted in green while the remaining seven disks remained white. Subjects were requested to indicate whether the highlighted disk was a target or a distractor by pressing one of two buttons on a button box (duration of response phase, $2 \mathrm{~s}$ ). The disk trajectories for each attentional tracking trial in each experiment were precomputed and the same trajectories were used for all subjects. Attentional tracking trials were presented in random order to each subject in each experiment. 
We did not monitor eye movements in the current study during visual attentional tracking. For a previous study (Frank et al., 2016a) using a similar visual attentional tracking paradigm as in the current study, we monitored eye movements during a psychophysical practice session outside the scanner. We observed that subjects maintained fixation very well during visual attentional tracking and that fixation quality was highly similar between different visual attentional tracking loads (Frank et al., 2016a). Furthermore, the greater the attentional load on the visual tracking task (i.e., the more disks had to be tracked as targets), the more would pursuit eye movements following one of the targets make it difficult to simultaneously follow other targets, because all stimuli move randomly in different directions. Therefore, visual attentional tracking is best performed when subjects maintain central fixation and try to follow the targets only with their attention. We therefore conclude it is unlikely that our fMRI or fMRS results were affected by eye movements.

$f M R I$ experiment with attentional tracking. In the fMRI experiment with attentional tracking, the response phase after each tracking trial was followed by feedback presented for $2 \mathrm{~s}$ (consisting of the fixation spot turning green or red indicating a correct or incorrect response). Each 20-s-long attentional tracking trial was always followed by a 20 -s-long blank baseline trial with central fixation. BOLD activation was measured in low-load (tracking one disk and two disks) and high-load (tracking three and four disks) attentional tracking conditions. There were 8 trials for each of the four tracking conditions within each fMRI run, totaling 32 attentional tracking trials. Attentional tracking trials were presented in random order. Each fMRI run was $21 \mathrm{~min}$ long, and each subject performed two fMRI runs.

$f M R S$ experiment with attentional tracking. In the fMRS experiment with attentional tracking the response phase was followed by a 2-s-long break with central fixation. No feedback was presented to avoid any confounds between the fMRS signal and feedback-related brain activation. Each tracking trial was immediately followed by a new tracking trial. Each fMRS run contained successive point-resolved spectroscopy (PRESS) and MescherGarwood PRESS (MEGA-PRESS) scans to measure the concentrations of Glx and GABA, respectively, in PIVC of the right hemisphere (see below). The attentional load remained identical within each fMRS run. Two separate fMRS runs were conducted for the low- and highattentional load conditions. The run order and the order of PRESS and MEGA-PRESS scans within a run were counterbalanced across subjects. Track 2 and track 4 served as the low- and high-attentional load conditions, respectively. Subjects completed a total of 60 attentional tracking trials within each fMRS run (run length, $20 \mathrm{~min}$ ). For female subjects not using contraception, the fMRS measurements were only conducted during the female follicular phase to avoid any confounds of the fMRS signal with the menstrual cycle (Duncan et al., 2014; De Bondt et al., 2015).

$f M R I$ experiment with attentional tracking and CVS. In this experiment, subjects performed visual attentional tracking during simultaneous CVS. There was a total of three fMRI runs for different experimental conditions. In a first fMRI run, CVS was conducted while subjects kept their eyes closed and did not perform any particular task (CVS-only condition). This condition served as a control to measure the BOLD response in PIVC to vestibular cues without any visual input. Previous results in human subjects (Deutschländer et al., 2002) showed that vestibular responses in PIVC and other areas of the vestibular cortex are strongest during CVS with eyes closed. Thus, this condition served to measure the BOLD response to vestibular cues under optimized stimulation conditions, similar to the CVS localizer experiment (see below).

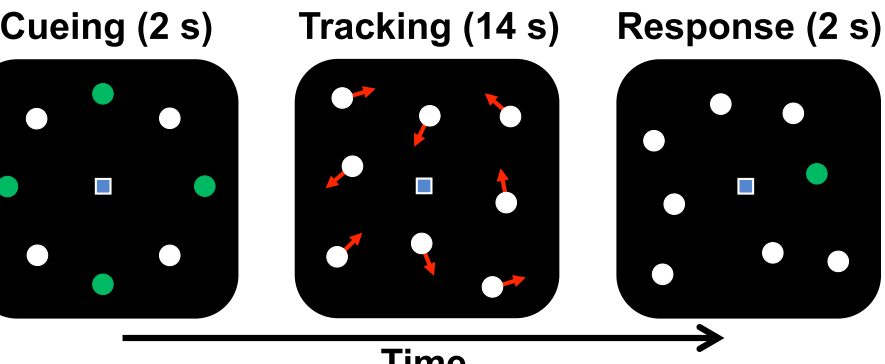

Time
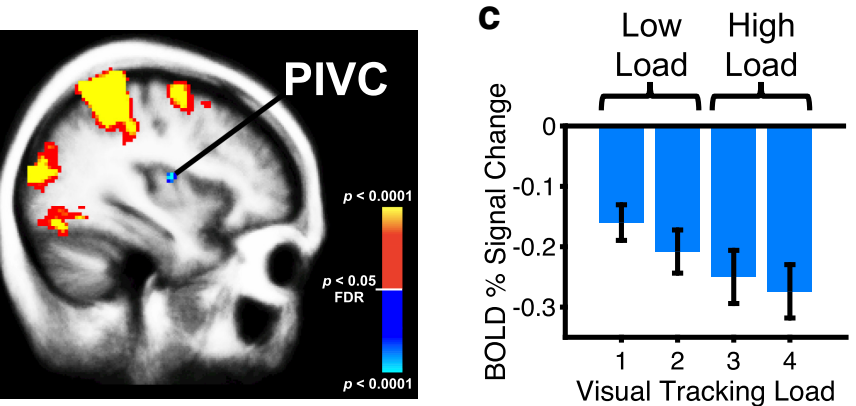

Visual Tracking Load

Figure 1. Cross-modal suppression of the PIVC during visual attentional tracking. $\boldsymbol{a}$, Example trial of the visual attenfigh-visual attentional load condition. During tracking, all disks were white and physically indistinguishable

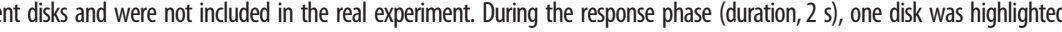
each tracking condition. PIVC was independently defined by using average anatomic coordinates (taken from Frank et al., 2016b). Zero on the $y$-axis corresponds to BOLD activation during central fixation on an otherwise blank screen.

To induce vestibular responses by means of CVS, it is necessary to conduct CVS for longer periods of time, typically for $\sim 60 \mathrm{~s}$ (Deutschländer et al., 2002; Dieterich et al., 2003; Indovina et al., 2005; Roberts et al., 2017). Previous studies measuring brain activation during CVS by means of fMRI in human subjects noted that vestibular activation tended to increase in magnitude over time (Frank et al., 2014), reaching a pronounced magnitude $\sim 30 \mathrm{~s}$ after onset of CVS (Roberts et al., 2017), which also corresponds to the time point at which subjects experience noticeable vestibular sensations (Suzuki et al., 2001). In a second fMRI run, CVS was paired with the low-visual attentional load condition (track 2). In a third fMRI run, CVS was paired with the high-visual attentional load condition (track 4). The fMRI runs were conducted in counterbalanced order across subjects. Each fMRI run consisted of six CVS trials (three with hot left and cold right, vice versa for the other three trials) and was $\sim 14 \mathrm{~min}$ in duration. Each CVS trial was $70 \mathrm{~s}$ long and was always followed by a 70 -slong baseline with warm thermal stimulation in both ears. During baseline, subjects either kept their eyes closed in the CVS-only condition or maintained central fixation in the low- and high-visual attentional load conditions. CVS was conducted as in the CVS localizer experiment (see below). On each CVS trial, temperatures were switched to hot left and cold right or cold left and hot right. The first $10 \mathrm{~s}$ of each CVS and baseline trials served as an on-ramp until temperatures reached steady-state levels (Frank and Greenlee, 2014). During the following $60 \mathrm{~s}$ with CVS, subjects performed three successive attentional tracking trials ( $20 \mathrm{~s}$ for each tracking trial) in a similar fashion as in the attentional tracking experiment with fMRS. No feedback about subjects' performance was provided. 
fMRI CVS localizer experiment. Bithermal CVS was conducted with an MRI-safe CVS device (Frank and Greenlee, 2014). The ear canals were stimulated with hot $\left(\sim 45^{\circ} \mathrm{C}\right)$ on the left side and cold $\left(\sim 22^{\circ} \mathrm{C}\right)$ on the right side (or vice versa). Warm in both ear canals $\left(\sim 36.5^{\circ} \mathrm{C}\right)$ served as baseline. Circulating water was used as the medium for different temperatures. Hot and cold water was stored in two plastic barrels in the scanner control room. Pumps in each barrel transported the water via waveguide tubes to the subject in the scanner. The tempered water circulated through small glass pods installed in the MRI headphone system and was returned to a collecting plastic barrel in the control room via separate tubes. Thus, CVS was performed by altering the temperature of the glass pods while the water remained inside the closed-loop system.

For fMRI, CVS alternated with warm stimulation for baseline. Each trial was $60 \mathrm{~s}$ long. The first $10 \mathrm{~s}$ served as on-ramp until temperatures reached steady-state levels. During the experiment, subjects kept their eyes closed and did not perform any particular task. Overall, 20 trials (5 for hot left and cold right, 5 for cold left and hot right, and 10 for warm) were conducted in a single fMRI run (run duration, $20 \mathrm{~min}$ ). The order of hot and cold on left or right sides was randomized for each subject.

After scanning, subjects completed a questionnaire and reported on their overall sensations during CVS (Frank et al., 2016b). The results of this questionnaire showed that each of 20 subjects sensed self-motion during CVS. The majority of subjects $(n=11)$ sensed self-motion as rotation, sometimes accompanied by sensations of sway in another 7 subjects. Two subjects only noticed sensations of sway. Twelve subjects reported that rotation occurred in the yaw plane, 2 subjects reported rotation in the roll plane, 3 subjects reported a combination of yaw and roll, and 2 subjects reported a combination of yaw and pitch. One subject reported a combination of roll and pitch. Eleven subjects sensed selfmotion of the head, while 9 subjects sensed self-motion of the whole body. None of the subjects reported having fallen asleep during CVS. None experienced any motion sickness or discomfort. Compared with conventional CVS using caloric irrigation of the ear canals with tempered water or gas, our CVS device only applied temperature cues to the ear canals while the water used for stimulation remained inside a closedloop system. With this setup, subjects experienced milder CVS than with caloric irrigation using direct injection of a tempered medium such as water or gas into the ear canal. An advantage of our approach is that negative side effects such as motion sickness that may occur during CVS are largely avoided and subjects primarily experience vestibular sensations of self-motion (e.g., rotation in the yaw plane; see Frank et al., $2014,2016 a, b, 2020)$. Other imaging studies using caloric irrigation also reported that very few or none of the subjects experienced motion sickness during CVS (Suzuki et al., 2001; Deutschländer et al., 2002; Indovina et al., 2005; Roberts et al., 2017). Our CVS approach may lead to a slightly lower intensity of vestibular stimulation compared with conventional caloric irrigation.

The purpose of the CVS localizer experiment was to define PIVC in the right hemisphere by means of stronger activation during CVS compared with baseline. Since only the right hemisphere was of interest for this study, this localizer could have been limited to a CVS condition with right hot and left cold. However, since some of the subjects in this study completed the localizer with both hot left and cold right as well as cold left and hot right CVS conditions for the purpose of a previous study (Frank et al., 2020; see Subjects), we decided to use the same experimental protocol for new subjects.

fMRI temperature control experiment. An fMRI control experiment was conducted to exclude activations from the definition of PIVC that may have arisen because of different temperature sensations rather than vestibular sensations (Frank et al., 2020). For this experiment, the same stimulation as for CVS was used, but the stimulation pods were attached to the pinna rather than inserted into the ear canals. Thus, subjects sensed the different temperatures but did not receive any vestibular cues. One fMRI run was conducted, following the same protocol as in the CVS experiment. After the experiment, all 20 subjects reported that they sensed the different temperatures, but none reported any sensations of self-motion. The control experiment was conducted before CVS.

$f M R I$ visual motion localizer experiment. An additional control experiment was conducted to confine the definition of PIVC to voxels primarily responsive to vestibular rather than visual cues. Previous results showed that a visual-vestibular area, referred to as the posterior insular cortex (PIC), is located in close proximity, just posterior to PIVC (Frank et al., 2014, 2016b; Frank and Greenlee, 2018). In contrast to PIVC, PIC is strongly activated during visual attentional tracking (Frank et al., 2016a, 2020). Since the major focus of this study was to investigate the suppression of PIVC during visual attentional tracking, we aimed to restrict the acquisition of the fMRS signal to PIVC only. Therefore, a functional localizer scan for PIC was conducted as described previously (Frank et al., 2014, 2016a,b, 2020). In short, 12-s-long blocks with translational visual motion of small white dots in different directions alternated with 12-s-long blocks of static white dots for baseline. There was a total of 24 blocks with visual motion and 24 baseline blocks. Each block with visual motion was followed by a baseline block. Subjects performed a dimming detection task on the central fixation spot. One run $(\sim 9.6 \mathrm{~min})$ was conducted.

Stimulus presentation. Stimuli were computed and presented using Psychtoolbox (Brainard, 1997; Pelli, 1997) running in MATLAB (MathWorks). Stimuli were projected onto a translucent screen at the back of the scanner bore. Subjects viewed the screen via a headcoilmounted mirror.

Imaging parameters. The fMRI data for the visual attentional tracking experiment without CVS were collected on a Intera Achieva 3 tesla MRI scanner (Philips) using a 32-channel head coil. Functional MRI data were acquired with a T2*-weighted echoplanar imaging $(\mathrm{EPI})$ sequence [repetition time $(\mathrm{TR})=2 \mathrm{~s}$; echo time $(\mathrm{TE})=35 \mathrm{~ms}$; flip angle $(\mathrm{FA})=90^{\circ}$; in-plane acquisition matrix $(\mathrm{AM})=80 \times 80 ; 34$ axial slices; voxel size $=3 \times 3 \times 3.5 \mathrm{~mm}$; no interslice gap]. A high-resolution anatomic scan of each subject's brain was acquired with a magnetization prepared rapid gradient echo (MPRAGE) sequence $\left(\mathrm{TR}=2.25 \mathrm{~s} ; \mathrm{TE}=2.6 \mathrm{~ms} ; \mathrm{FA}=8^{\circ} ; \mathrm{AM}=256 \times 256 ; 220\right.$ sagittal slices; voxel size $=1 \times 1 \times 1 \mathrm{~mm}$; interslice gap $=0.5 \mathrm{~mm}$ ).

All other imaging data were collected on a 3 tesla MAGNETOM Prisma MRI scanner (Siemens Healthcare). The fMRI data for the visual attentional tracking experiment with CVS and the localizer experiments were acquired with a 20 -channel head coil and a T2*-weighted EPI sequence $\left(\mathrm{TR}=2 \mathrm{~s} ; \mathrm{TE}=30 \mathrm{~ms} ; \mathrm{FA}=90^{\circ} ; \mathrm{AM}=64 \times 64 ; 32\right.$ axial slices; voxel size $=3 \times 3 \times 3 \mathrm{~mm}$; interslice gap $=0.5 \mathrm{~mm}$ ). For each subject, a high-resolution anatomic scan of the brain was collected with a 64-channel head/neck coil using an MPRAGE sequence $(\mathrm{TR}=2.3 \mathrm{~s}$; $\mathrm{TE}=2.32 \mathrm{~ms} ; \mathrm{FA}=8^{\circ} ; \quad \mathrm{AM}=256 \times 256 ; 192$ sagittal slices; voxel size $=0.9 \times 0.9 \times 0.9 \mathrm{~mm}$; interslice gap $=0.45 \mathrm{~mm})$.

Single-voxel proton $\left({ }^{1} \mathrm{H}\right)$ MR spectroscopy was conducted with the 64-channel head/neck coil. The concentration of Glx was measured with a PRESS-sequence $\left(\mathrm{TR}=3 \mathrm{~s}\right.$; $\mathrm{TE}=30 \mathrm{~ms} ; \mathrm{FA}=90^{\circ}$; number of averages $=128$; scan time $=384 \mathrm{~s}$; Bottomley, 1984, 1987). The concentration of GABA was measured with a MEGA-PRESS sequence $\left(\mathrm{TR}=1.5 \mathrm{~s} ; \mathrm{TE}=68 \mathrm{~ms} ; \mathrm{FA}=90^{\circ}\right.$; number of averages $=256$; scan time $=774$ s; Mescher et al., 1996, 1998). The scanning parameters, including the number of averages, for PRESS and MEGA-PRESS were adapted from previous studies (Shibata et al., 2017; Bang et al., 2018). A frequency-selective, single-band Gauss pulse was used to saturate the $\beta-\mathrm{CH}_{2}$ signal at $1.94 \mathrm{ppm}$ and to refocus the J evolution of the triplet $\gamma-\mathrm{CH}_{2}$ resonance of GABA at $3 \mathrm{ppm}$ ("Edit On"). The very same Gauss pulse was used to irradiate the opposite part of the spectrum at $7.46 \mathrm{ppm}$ ("Edit Off"). The difference spectra were produced by subtracting the Edit Off from the Edit On spectra. For both PRESS and MEGA-PRESS sequences, WET (water suppression enhanced through T1 effects) water suppression was used (Ogg et al., 1994).

The fMRS data were acquired within a single voxel $(2.5 \times 2.5 \times$ $2.5 \mathrm{~cm}$ ) that was placed manually at the location of PIVC in the mid-posterior Sylvian fissure. To determine the location of PIVC in individual subjects, we used the fMRI activation of PIVC during CVS (see Fig. 3c). The voxel in PIVC was placed with respect to the location of the visualvestibular area PIC that is located at a more posterior location in the Sylvian fissure (Frank et al., 2016b). Any overlap between the voxel centered on PIVC and area PIC was avoided. The percentage of overlap between the fMRS voxel and PIC was negligible: across subjects, the mean \pm SE percentage of brain tissue corresponding to PIC contained 
within the fMRS voxel was $1.39 \pm 1.16 \%$. Three short, high-resolution anatomic scans (for coronal, sagittal, and transverse planes, respectively) were conducted to support the fMRS voxel placement with the following scanning parameters: coronal plane $\left(\mathrm{TR}=0.25 \mathrm{~s} ; \mathrm{TE}=2.46 \mathrm{~ms} ; \mathrm{FA}=70^{\circ}\right.$; $\mathrm{AM}=288 \times 288 ; 35$ slices; voxel size $=0.8 \times 0.8 \times 4.0 \mathrm{~mm}$; interslice gap $=1.20 \mathrm{~mm})$, sagittal plane $\left(\mathrm{TR}=0.19 \mathrm{~s} ; \mathrm{TE}=2.46 \mathrm{~ms} ; \mathrm{FA}=70^{\circ}\right.$; $\mathrm{AM}=288 \times 288 ; 25$ slices; voxel size $=0.8 \times 0.8 \times 4.0 \mathrm{~mm}$; interslice gap $=1.20 \mathrm{~mm})$, transverse plane $\left(\mathrm{TR}=0.19 \mathrm{~s} ; \mathrm{TE}=2.46 \mathrm{~ms} ; \mathrm{FA}=70^{\circ}\right.$; $\mathrm{AM}=288 \times 288 ; 27$ slices; voxel size $=0.8 \times 0.8 \times 4.0 \mathrm{~mm}$; interslice gap $=1.20 \mathrm{~mm}$ ). During voxel placement non-neural tissue containing lipids was avoided. Automatic shimming was applied before PRESS and MEGA-PRESS fMRS acquisitions, followed by manual shimming if necessary. The shim values for each subject and each fMRS acquisition were kept below a full-width at half-maximum (FWHM) of $20 \mathrm{~Hz}$. The mean ( \pm SE) shim values across subjects for each experimental condition and fMRS acquisition were as follows: $15.5 \pm 0.39 \mathrm{~Hz}$ (low load, PRESS), $15.6 \pm 0.38 \mathrm{~Hz}$ (high load, PRESS), $15.6 \pm 0.41 \mathrm{~Hz}$ (low load, MEGAPRESS), $15.7 \pm 0.42 \mathrm{~Hz}$ (high load, MEGA-PRESS). Post hoc Wilcoxon signed-rank tests did not show any significant differences in shim values between different experimental conditions and fMRS acquisitions (all $p>0.05$ ).

MRI analysis. Anatomical and functional MRI data were analyzed with the Freesurfer software package (Martinos Center for Biomedical Imaging, Charlestown, MA). Each subject's high-resolution anatomic scan of the brain was reconstructed and inflated (Dale et al., 1999; Fischl et al., 1999). Volume fractions for the fMRS voxel in PIVC were computed by using the automated anatomic segmentation of the brain into gray matter (GM), white matter (WM), and cerebrospinal fluid (CSF) during reconstruction. Functional MRI data were motion corrected; coregistered to the reconstructed anatomic brain; smoothed with a threedimensional Gaussian kernel (FWHM $=5 \mathrm{~mm}$ ), except the analysis of the thalamus for which no smoothing was performed (see below); and intensity normalized.

Preprocessed functional MRI data were analyzed using a general linear model (GLM) approach. The BOLD response was modeled with the SPM hemodynamic response function. Each GLM contained a linear scanner drift predictor and motion correction parameters as regressorsof-no-interest.

The GLM of the visual attentional tracking experiment without CVS contained four regressors-of-interest. These regressors included trials for each of the four tracking conditions (corresponding to tracking one to four disks). All trials, regardless of correct or incorrect subject response, were modeled. Furthermore, there was a regressor for blank baseline trials and two regressors for the cueing and response/feedback phases at the beginning and at the end of the tracking trials, respectively. Activation in the high-attentional load conditions (corresponding to tracking three and four disks) was contrasted with activation in the lowattentional load conditions (corresponding to tracking one and two disks; Fig. 1b). Furthermore, deactivation in right PIVC was computed as the BOLD percentage signal change from fixation baseline for each tracking load (Fig. 1c). To this aim, PIVC was defined in each subject from this group by placing a sphere with a $5 \mathrm{~mm}$ radius at the average location of PIVC, using previously published coordinates (in Talairach space: $x=40, y=-14, z=18$; Frank et al., 2016b). This coordinate-based definition of PIVC is highly consistent with the mean location of PIVC across subjects in the second experiment for which PIVC was defined functionally by means of CVS (see Fig. $3 a$ ). Furthermore, the center of the location of PIVC, as defined by means of CVS, is highly consistent across subjects (see Fig. $3 b$ ). Therefore, although the coordinate-based definition of PIVC in the first experiment is a limitation, this definition is a valid approximation of the location of PIVC in individual subjects.

Each run of the fMRI experiment with visual attentional tracking and simultaneous CVS was analyzed by carrying out a separate GLM. Each GLM contained three regressors-of-interest. Two regressors were included for CVS with hot left and cold right and cold left and hot right, respectively. A third regressor was used for warm baseline. Each regressor included the central $60 \mathrm{~s}$ of each CVS or baseline trial after the 10-slong on-ramp with temperature changes. For a control analysis of the CVS-only condition, the BOLD activation in PIVC during the 60-s-long
CVS was separated into three blocks (the first $20 \mathrm{~s}$, the interim $20 \mathrm{~s}$, and the final $20 \mathrm{~s}$ of CVS) to test whether BOLD activation in PIVC changed in magnitude over the course of CVS. The activation of right PIVC (as defined in the CVS localizer experiment; see below) was computed as the BOLD percentage signal change from warm baseline (either with eyes closed in the CVS-only control condition or with central fixation in the visual attentional tracking conditions; see Fig. 4). A similar analysis was conducted for the thalamus (see below), except that unsmoothed fMRI scans were submitted to the GLM analysis to minimize partial volume effects (see Fig. 5).

Region of interest definition. The following three regions of interest (ROIs) were defined: PIVC, PIC, and a subset of thalamic nuclei with strong connectivity with PIVC. PIVC was defined using the CVS and temperature control experiments. The activations in these experiments were analyzed by GLMs with three separate regressors of interest for hot left and cold right, cold left and hot right, and warm both sides. The BOLD response was estimated for the central $50 \mathrm{~s}$ of each trial following the 10-s-long on-ramp with temperature changes. In both experiments, activation during CVS (corresponding to hot left and cold right and cold left and hot right) was contrasted with baseline (corresponding to warm in both ears). PIVC in the right hemisphere was defined by stronger activation during CVS at a threshold of $p<0.05$, FDR corrected (see Fig. $3 a$, group analysis, $c$, sample subject). The same threshold was used for activation in the temperature control experiment. Overlapping voxels between the CVS and temperature control experiments were removed from the definition of PIVC. The mean $( \pm$ SE) percentage of voxels of PIVC overlapping between CVS and temperature control was $6.18 \pm 9.27 \%$. The mean $( \pm S E)$ Talairach coordinates of PIVC in the right hemisphere across subjects were $x=39 \pm 1, y=-11 \pm 1, z=15 \pm 1$. Across subjects, PIVC had a mean size $( \pm$ SE) of $71 \pm 11$ voxels in functional space $(3 \times 3 \times 3 \mathrm{~mm})$. See Figure $3 b$ for the anatomic location of the center of PIVC in each subject.

Furthermore, PIVC was separated from PIC, which is located at a more posterior site in the Sylvian fissure (Frank et al., 2014, 2016b; Frank and Greenlee, 2018). Area PIC was defined by using activation in the visual motion localizer. The GLM of the visual motion localizer included two regressors of interest for the visual motion and static baseline conditions. Area PIC was defined by contrasting activation during visual motion with static baseline at a threshold of $p<0.05$ FDR corrected. The mean $( \pm \mathrm{SE})$ Talairach coordinates of PIC in the right hemisphere across subjects were $x=49 \pm 2, y=-29 \pm 1, z=22 \pm 1$. Across subjects PIC had a mean size ( \pm SE) of $58 \pm 7$ voxels in functional space $(3 \times 3 \times 3 \mathrm{~mm})$. No overlap between voxels corresponding to PIVC and voxels corresponding to PIC was observed in any subject.

The thalamus ROI was defined using an anatomic atlas of the thalamic nuclei in the human brain (Krauth et al., 2010), which was nonlinearly transformed into the individual structural space and from there mapped into the individual functional space. Using diffusion-weighted imaging and probabilistic fiber tracking in human subjects, a previous study found that PIVC exhibited the strongest structural connectivity with the ventral anterior and the ventral posterior lateral thalamic nuclei (Wirth et al., 2018). Therefore, we used these thalamic nuclei for the analysis of activation in the experiment with visual attentional tracking and simultaneous CVS. Since the vestibular projections from the vestibular nuclei in the brainstem are bilateral (Lopez and Blanke, 2011), the thalamic nuclei were combined across left and right sides. Furthermore, the results were combined across nuclei to increase the signal-to-noise ratio of the analysis, given the small size of each nucleus. The mean $( \pm S E)$ Talairach coordinates of the included thalamic nuclei across subjects were as follows: ventral anterior nucleus: left: $x=-11 \pm 0.03, y=$ $-6 \pm 0.01, z=3 \pm 0.03$; right: $x=12 \pm 0.03, y=-6 \pm 0.02, z=4 \pm 0.03$; ventral posterior lateral nucleus: left: $x=-18 \pm 0.03, y=-19 \pm 0.02$, $z=6 \pm 0.02$; right: $x=19 \pm 0.03, y=-19 \pm 0.02, z=6 \pm 0.02$. Combined across nuclei and sides, the mean $( \pm \mathrm{SE})$ size of the final thalamic ROI across subjects was $18 \pm 1$ voxels in functional space $(3 \times 3 \times 3 \mathrm{~mm}$; see Fig. $5 a$ for the location in a sample subject).

MRS analysis. The fMRS PRESS and MEGA-PRESS spectra in PIVC during low and high loads of visual attentional tracking are shown for 
a

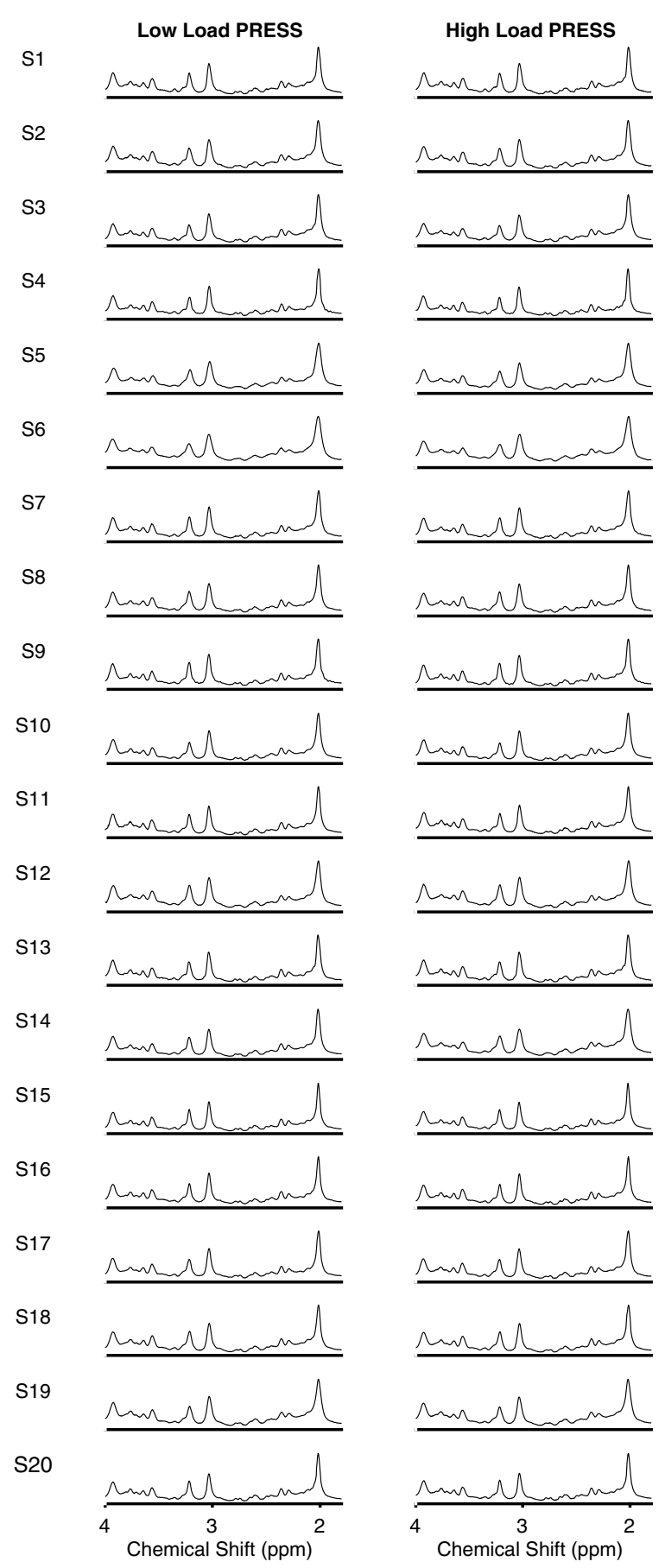

C

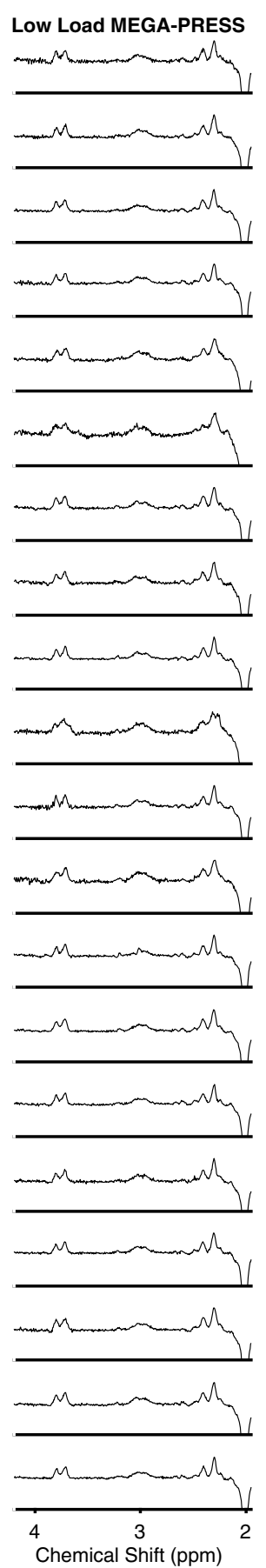

d

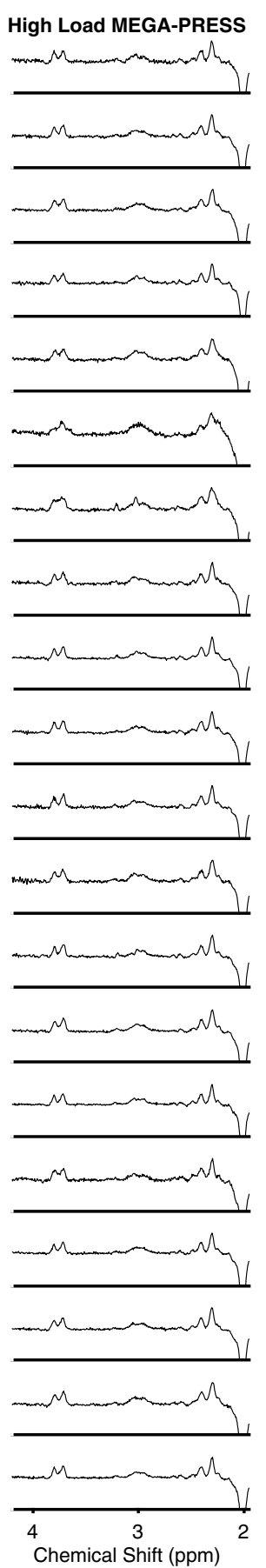

Figure 2. fMRS spectra in right PIVC during visual attentional tracking. $\boldsymbol{a}$, Spectra during the low-visual attentional load condition taken from the PRESS scan. The Glx peak is seen at 2.1$2.5 \mathrm{ppm}$. Each row shows the result from a different subject (S1-S20). $\boldsymbol{b}$, Same as $\boldsymbol{a}$ but for the high-visual attentional load condition. $\boldsymbol{c}$, Spectra during the low-visual attentional load condition taken from the MEGA-PRESS difference scan. The GABA peak is seen at 3 ppm. $\boldsymbol{d}$, Same as $\boldsymbol{c}$ but for the high-visual attentional load condition.

each subject in Figure 2. The metabolite intensities of the fMRS spectra were fitted with the LC model (Provencher, 1993, 2001) in the chemical shift range between 0.2 and $4.0 \mathrm{ppm}$ for PRESS and 1.95 and $4.2 \mathrm{ppm}$ for MEGA-PRESS. The metabolite intensities were fitted to a linear combination of spectra of individual metabolites derived from an imported metabolite basis set (Provencher, 1993, 2001). The CramerRao Lower bounds (CRLB) indicate the reliability of quantification of Glx and GABA. We used a commonly accepted criterion of $20 \%$ to reject low-quality results. None of the Glx or GABA results in any experimental condition had to be rejected under this criterion. For Glx (PRESS), the mean ( \pm SE) CRLB percentage across subjects in PIVC were $4.85 \pm 0.11 \%$ in the low-load and $5.20 \pm 0.09 \%$ in the high-load visual attentional tracking conditions. For GABA (MEGAPRESS), the corresponding values were $4.70 \pm 0.16 \%$ in the low-load and $4.55 \pm 0.11 \%$ in the high-load tracking conditions. For each experimental condition and subject, the concentration of Glx, measured by PRESS, was normalized to the concentration of total creatine [ $\mathrm{tCr}$ (corresponding to creatine-phosphocreatine)] taken from the same 
scan (Fig. 3d). Creatine is a measure of cellular integrity; it is relatively stable within each tissue type and a standard control metabolite for the normalization of other metabolite concentrations (Duncan et al., 2014; Stagg, 2014). The concentrations of tCr did not differ significantly between the low- and high-visual attentional load conditions across subjects (paired-sample $t$ test, $\left.t_{(19)}=-0.21, p=0.83, d=-0.05\right)$. For a control analysis, Glx was normalized to $N$-acetylaspartate (NAA). NAA is a marker of neuronal density and mitochondrial function and another standard control metabolite used for normalization (Duncan et al., 2014; Stagg, 2014). The concentrations of NAA did not differ significantly between the low- and high-visual attentional load conditions across subjects $\left(t_{(19)}=\right.$ $-0.56, p=0.58, d=-0.13)$. Since no creatine peak is acquired, the concentration of GABA in the MEGAPRESS difference spectrum was normalized to the concentration of NAA, taken from the same scan and MEGA-PRESS difference spectrum (Fig. 3e). The concentrations of NAA did not differ significantly between the low- and high-visual attentional load conditions across subjects $\left(t_{(19)}=-0.33, p=0.74, d=\right.$ $-0.07)$. The mean volume fractions $( \pm S E)$ of $G M$, $\mathrm{WM}$, and CSF of the fMRS voxel centered on right PIVC across subjects were $53.6 \pm 2.26 \%, 28.9 \pm 2.93 \%$, and $17.5 \pm 3.38 \%$, respectively. For a control analysis, the concentrations of Glx, GABA, tCr, and NAA were corrected for the contribution of different contents of tissue within the PIVC voxel (partial volume effect) before normalization. Following previous descriptions (Kolasinski et al., 2017), the concentrations of Glx and GABA were corrected for the proportion of gray matter within the voxel by dividing by $[\mathrm{GM} /(\mathrm{GM}+\mathrm{WM}$ $+\mathrm{CSF})]$. The concentrations of tCR and NAA were corrected for the proportion of total brain volume within the voxel by dividing by $[(\mathrm{GM}+\mathrm{WM}) /(\mathrm{GM}+$ $\mathrm{WM}+\mathrm{CSF}$ )]. Similar to the primary analysis (see above), the concentrations of the control metabolites did not differ significantly between the low- and highvisual attentional load conditions after partial volume correction (tCr from PRESS: $t_{(19)}=-0.52, p=0.61, d=$ -0.12 ; NAA from PRESS: $t_{(19)}=-0.46, p=0.65, d=$ -0.10 ; NAA from MEGA-PRESS: $t_{(19)}=-0.66, p=0.52, d=-0.15$ ).

Statistical analysis. The sample size of this study was determined based on previous studies (Hu et al., 2013; Frank et al., 2016a, 2020; Shibata et al., 2017; Bang et al., 2018; Martínez-Maestro et al., 2019; Boillat et al., 2020) and the recommendations of a meta-analysis (Duncan et al., 2014). Data were analyzed using parametric statistics after confirmation of the assumption of normality. Since ShapiroWilk tests showed that the assumption of normality was violated for the behavioral accuracy results in the attentional tracking experiments, each subject's accuracy $(p)$ was arcsin square root transformed using the following formula: $p^{\prime}=\arcsin (\sqrt{ } p)$. Post hoc Shapiro-Wilk tests showed that the transformed accuracy results $\left(p^{\prime}\right)$ did not deviate from normality in any experiment. Since the assumption of normality was also violated for the shim value results (as shown by significant Shapiro-Wilk tests), nonparametric Wilcoxon signed-rank tests were used instead of ANOVA. For all statistical tests, the two-tailed $\alpha$-level was set to 0.05 . Partial $\eta^{2}$, Cohen's $d$, and Pearson's $r$ values are reported as measures of effect size for ANOVA, $t$ test, and Pearson correlation, respectively. For the statistical comparison between Glx and GABA, the normalized metabolite concentrations of Glx and GABA were separately $z$ scored across visual attentional load conditions before submitting them to an ANOVA. One subject in the third experiment exhibited a BOLD percentage signal change in PIVC during the CVS-only condition that was more than 2 SDs away from the group mean. This subject was excluded from the analysis of this condition. b

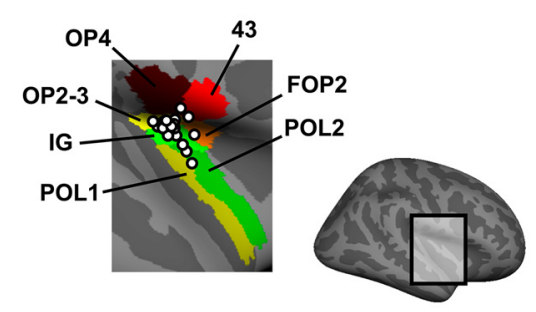

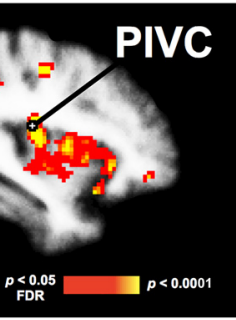
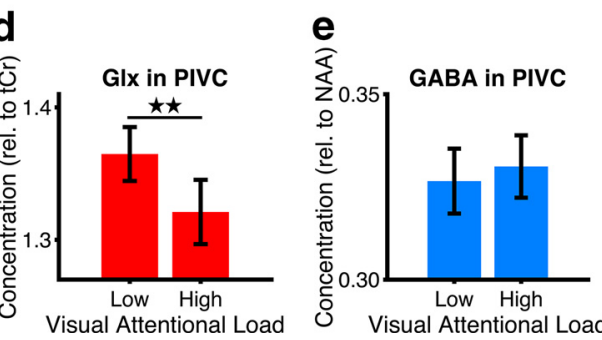
andersis for a total of 20 subjects overlaid on a template brain (right hemi(a) of visual attentional tracking (corresponding to tracking two and four disks, respectively) for 20 subjects. from the same scan. $\boldsymbol{e}$, Same as $\boldsymbol{d}$ but for GABA. The concentrations of GABA were measured by a MEGA-PRESS scan and are relative to NAA, a control metabolite taken from the same scan. ${ }^{* *} p<0.01$.

\section{Results}

Figure $1 b$ shows differences in BOLD signal between conditions of high and low visual attentional loads on the tracking task in the first experiment. This contrast revealed activations in the frontoparietal attention network and in the occipital cortex (see also Culham et al., 2001; Frank et al., 2016a) and deactivation in PIVC. A post hoc ROI analysis showed that the BOLD signal in PIVC was significantly more negative for both the low-visual attentional load condition (corresponding to the mean BOLD signal across track 1 and track 2: $t_{(19)}=-6.06, p<0.001, d=$ -1.36 ) and the high-visual attentional load condition (corresponding to the mean BOLD signal across track 3 and track 4 : $\left.t_{(19)}=-6.22, p<0.001, d=-1.39\right)$ compared with baseline (corresponding to activation during central fixation; Fig. 1c). Furthermore, the BOLD signal was significantly more negative in the high-load compared with the low-load condition $\left(t_{(19)}=\right.$ $-3.73, p=0.001, d=-0.83)$. These results agree with previous observations (Brandt et al., 1998; Kleinschmidt et al., 2002; Frank et al., 2016a, 2020) and suggest that greater visual attentional loads are associated with more pronounced suppression of PIVC.

Further clusters with deactivation during high compared with low attentional load were located in the cuneus/precuneus, inferior and superior frontal cortex, inferior insula, parahippocampal 

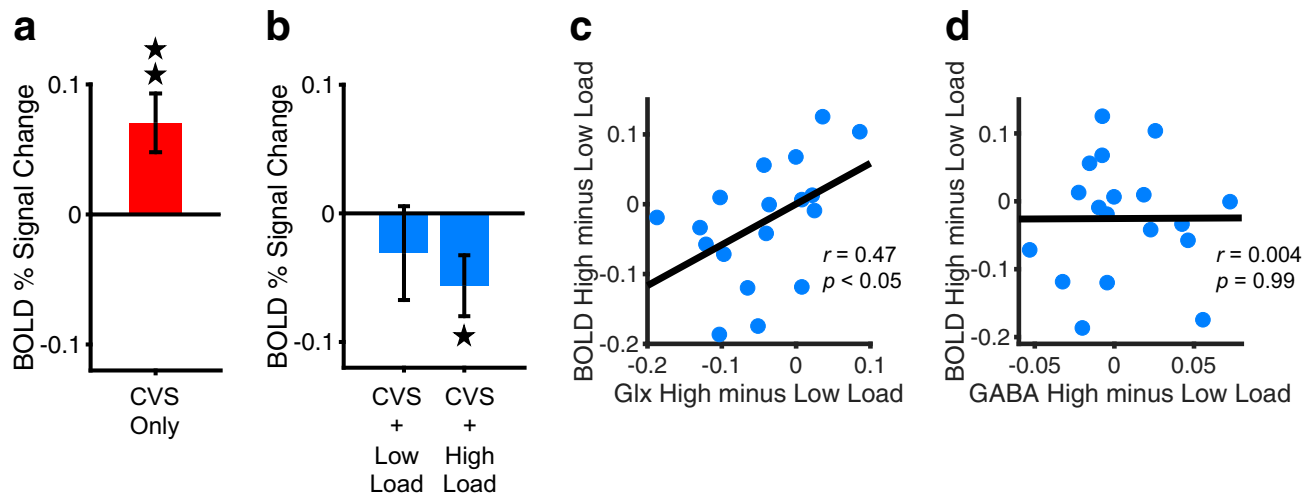

Figure 4. BOLD signal changes in PIVC during CVS combined with visual attentional tracking. $\boldsymbol{a}$, Control condition. Mean ( \pm SE) BOLD percentage signal change in right PIVC, while subjects ( $n=17$ from the fMRS experiment) received excitatory vestibular cues by means of CVS and kept their eyes closed. Zero on the $y$-axis corresponds to baseline with eyes closed and warm thermal stimulation in both ears. $\boldsymbol{b}$, Experimental conditions. Subjects received CVS and performed a visual tracking task with different attentional loads while maintaining central fixation. Low load corresponds to the track-two condition. High load corresponds to the track-four condition. Zero on the $y$-axis corresponds to baseline with central fixation and warm thermal stimulation in both ears. Results are shown for a total of 18 subjects. Otherwise, same as $\boldsymbol{a}$. c, Correlation between Glx and BOLD signal changes across subjects $(n=18)$. The Glx scores represent differences in the concentration of Glx in PIVC between high and low visual attentional loads from the fMRS experiment (high load minus low load; Fig. 3d). The BOLD scores represent differences in BOLD signal in PIVC between high and low visual attentional loads combined with CVS (high load minus low load, see $\boldsymbol{b}$ ). Each dot shows the result from a different subject. Subjects with a greater decrease in Glx from low to high load (corresponding to lower values on the $x$-axis) tended to show a larger increase in negative BOLD signal from low to high load (corresponding to lower values on the $y$-axis). $\boldsymbol{d}$, Correlation between GABA and BOLD across subjects $(n=18)$. Otherwise, same as $c .{ }^{*} p<0.05,{ }^{* *} p<0.01$.

cortex, posterior cingulate cortex, subparietal sulcus, and superior temporal sulcus. This set of regions resembles the results of a previous study using a similar visual attentional tracking paradigm in fMRI (Frank et al., 2016a) and overlaps with the default mode network that is deactivated when attention is focused on processing of external stimuli (Raichle, 2015). It is important to note that PIVC is not considered part of the default mode network (Raichle, 2015).

The mean $( \pm$ SE) accuracy on the tracking task was $89.7 \pm$ $0.03 \%$ in the low-load condition and $84.4 \pm 0.03 \%$ in the highload condition. This difference in accuracy between attentional load conditions was significant (paired-sample $t$ test on arcsintransformed tracking accuracy, low load vs high load: $t_{(19)}=3.51$, $p=0.002, d=0.78)$. Importantly, this decrease in visual tracking performance in the high-load condition compared with the lowload condition did not correlate significantly with the BOLD signal changes between these two conditions across subjects $(r=$ $-0.36, p=0.12)$. This indicates that the increase in negative BOLD in the high-load condition compared with the low-load condition was not associated with a decrease in tracking accuracy, similar to results of a previous study (Frank et al., 2016a).

The results of the second experiment using fMRS showed that the concentrations of Glx and GABA in PIVC changed differently from the low- to the high-load visual attentional tracking conditions (Fig. 3d,e). For statistical comparison, the concentrations of Glx and GABA were separately $z$-scored and a $2 \times 2$ repeated-measures ANOVA with the factors of visual attentional load (low, high) and metabolite (Glx, GABA) was conducted. There was a significant interaction between visual attentional load and metabolite $\left(F_{(1,19)}=4.43, p=0.0488\right.$, partial $\left.\eta^{2}=0.19\right)$, indicating that the concentration of Glx decreased from low to high visual attentional load while the concentration of GABA remained stable across load conditions (Fig. $3 d, e$ ). In support of these results, post hoc paired-sample $t$ tests showed a significant decrease of Glx in the high-load condition compared with the low-load condition $\left(t_{(19)}=-2.90, p=0.009, d=-0.65\right.$; Fig. $\left.3 d\right)$, whereas no such difference between attentional loads was observed for GABA $\left(t_{(19)}=0.55, p=0.59, d=0.12\right.$; Fig. $\left.3 e\right)$. There was no significant correlation across subjects between changes in Glx and GABA from low load to high load ( $r=-0.13, p=0.59)$.
Similar significant differences between high and low visual attentional loads were observed when Glx was normalized relative to NAA $\left(t_{(19)}=-2.98, p=0.008, d=-0.67\right)$, and when the concentrations of the metabolites within the fMRS voxel were corrected for partial volume effects (high load vs low load; Glx: $t_{(19)}$ $=-2.99, p=0.008, d=-0.67$; GABA: $t_{(19)}=0.76, p=0.46$, $d=0.17)$.

The mean ( $\pm \mathrm{SE})$ accuracy on the tracking task was $87.0 \pm 1.46 \%$ in the low-load condition and $77.0 \pm 3.42 \%$ in the high-load condition. This difference in accuracy between attentional load conditions was significant (paired-sample $t$ test on arcsin-transformed tracking accuracy, low load vs high load: $\left.t_{(19)}=4.15, p<0.001, d=0.93\right)$. However, the decrease of Glx during high load was not associated with a decrease in behavioral performance as there was no significant correlation between the decrease of Glx and the decrease of behavioral performance from the high-load condition to the low-load condition across subjects $(r=0.09, p=0.72)$. Similarly, there was no significant correlation between changes in GABA and tracking accuracy between the two attentional load conditions $(r=-0.36, p=0.12)$. Furthermore, tracking performance did not differ significantly between Glx and GABA measurements. A $2 \times 2$ repeated-measures ANOVA with the factors of visual attentional load (low, high) and metabolite (Glx, GABA) on tracking performance did not show any significant interaction between visual attentional load and metabolite $\left(F_{(1,19)}=0.13, p=0.72\right.$, partial $\left.\eta^{2}=0.007\right)$ or any significant main effect of metabolite $\left(F_{(1,19)}=1.11, p=0.31\right.$, partial $\left.\eta^{2}=0.06\right)$. The main effect of visual attentional load was significant $\left(F_{(1,19)}=8.77\right.$, $p=0.008$, partial $\left.\eta^{2}=0.32\right)$ because of the lower tracking accuracy in the high-load condition compared with the low-load condition (see above).

In a third experiment, we examined the relationship between the decrease of Glx during high loads of visual attentional tracking and BOLD signal changes. Figure $4 a$ shows the mean BOLD percentage signal change in right PIVC during the CVSonly control condition (while subjects kept their eyes closed). Compared with baseline (corresponding to the condition with eyes closed and warm thermal stimulation in both ears), the BOLD signal in right PIVC was significantly increased during CVS only $\left(t_{(16)}=3.12, p=0.007, d=0.76\right)$, indicating that 
excitatory vestibular cues activated this core area of the vestibular cortex (note that one subject was excluded as an outlier from this analysis; see Statistical analysis). Similar to the results of a previous study (Roberts et al., 2017), the BOLD response in PIVC tended to increase over time with CVS: although the BOLD signal was not significantly different from baseline during the first $20 \mathrm{~s}$ after CVS onset $\left(t_{(16)}=-0.06, p=0.95, d=\right.$ $-0.01)$, there was a significant increase in both the interim $20 \mathrm{~s}$ of CVS $\left(t_{(16)}=4.21, p<0.001, d=1.02\right)$ and the final $20 \mathrm{~s}$ of CVS $\left(t_{(16)}=2.22, p=0.04, d=0.54\right)$. These results suggest that the BOLD response in PIVC was not affected by potential adaptation effects during CVS.

Figure $4 b$ shows deactivation in right PIVC during CVS combined with low and high visual attentional tracking loads (corresponding to track 2 and track 4, respectively, while subjects kept their eyes open). Compared with baseline (corresponding to central fixation with warm thermal stimulation in both ears), the BOLD signal changes in PIVC during CVS combined with tracking were significantly lower only in the high-load tracking condition $\left(t_{(17)}=-2.37, p=0.03, d=-0.56\right)$ but not in the low-load tracking condition $\left(t_{(17)}=-0.85, p=0.41, d=-0.20\right)$. The BOLD signals in high and low load were not significantly different $\left(t_{(17)}=-1.24, p=0.23, d=-0.29\right)$.

Importantly, changes in the concentrations of Glx in the fMRS experiment (Fig. $3 d$ ) and in BOLD in the fMRI experiment with CVS (Fig. 4b) from low to high loads of visual attentional tracking were significantly correlated across subjects (Fig. 4c). Specifically, subjects with a greater decrease of Glx from low to high load tended to show a greater increase in negative BOLD signal from low to high load during CVS $(r=0.47, p=0.0485)$. No such association was observed for GABA and BOLD $(r=$ 0.004, $p=0.99$; Fig. $4 d$ ).

The mean $( \pm \mathrm{SE})$ accuracy on the tracking task was $92.2 \pm$ $0.03 \%$ in the low-load condition and $84.4 \pm 0.03 \%$ in the highload condition. This difference in accuracy between attentional load conditions was significant (paired-sample $t$ test on arcsintransformed tracking accuracy, low load vs high load: $t_{(17)}=5.40$, $p<0.001, d=1.27)$. However, similar to the results of the previous experiments (see above), this decrease in tracking performance from low to high load was not significantly correlated with BOLD signal differences between the two conditions across subjects $(r=-0.17, p=0.49)$.

Finally, to test whether the attentional suppression occurred cortically by suppressing PIVC or subcortically by suppressing core vestibular nuclei of the thalamus (or other major subcortical input to the thalamus such as the vestibular nuclei in the brainstem), we analyzed the BOLD signal in core vestibular nuclei of the thalamus during visual attentional tracking combined with CVS (Fig. 5). The results showed a significantly stronger BOLD signal during CVS compared with baseline (corresponding to warm thermal stimulation in both ears; $t_{(17)}=2.12, p=0.0488$, $d=0.50$; Fig. $5 b$ ), indicating that the thalamic nuclei exhibited an increased activation in response to the vestibular cues similar to that found in PIVC (Fig. 4a). Next, we compared activation during CVS combined with low and high loads on the visual attentional tracking task between the thalamic nuclei and PIVC. A $2 \times 2$ repeated-measures ANOVA with the factors of stimulation condition (CVS + low load, CVS + high load) and ROI (vestibular thalamic nuclei, PIVC) showed a significant main effect of ROI $\left(F_{(1,17)}=14.1, p=0.002\right.$, partial $\left.\eta^{2}=0.45\right)$, indicating that BOLD signal changes were significantly different between the vestibular thalamic nuclei (Fig. 5c) and PIVC (Fig. 4b) across stimulation conditions. There was no significant main effect of
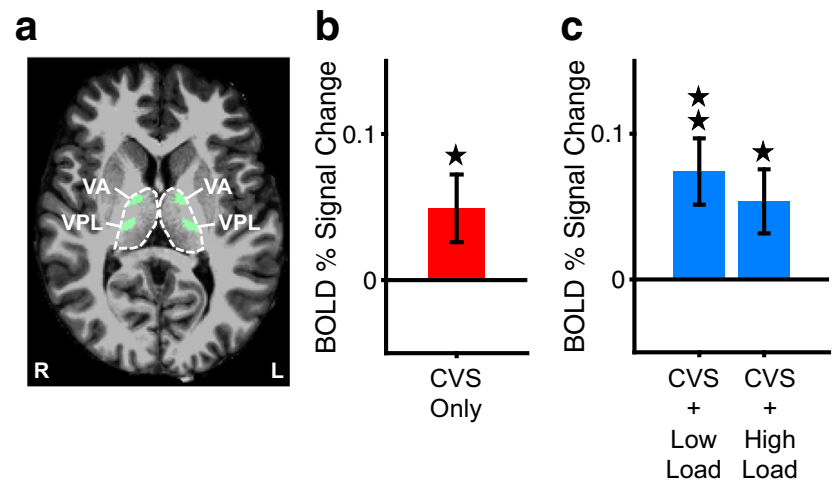

Figure 5. BOLD signal changes in vestibular thalamic nuclei during CVS-only or CVS combined with visual attentional tracking. $\boldsymbol{a}$, Location of the vestibular thalamic ROl consisting of the ventral anterior (VA) and ventral posterior lateral (VPL) thalamic nuclei, defined by using an anatomic atlas (Krauth et al., 2010), overlaid on the high-resolution anatomic brain of a sample subject ( $L$, left side; $R$, right side). Previous results (Wirth et al., 2018) showed that these vestibular nuclei of the thalamus have the strongest structural connectivity with PIVC in human subjects. Dashed lines show the outlines of the left and right thalamus as delineated by the anatomic segmentation of Freesurfer. $\boldsymbol{b}$, Mean ( \pm SE) BOLD percentage signal change in the vestibular thalamic ROI during CVS only. Otherwise, same as Figure $4 a$. c, Same as $\boldsymbol{b}$ but for BOLD percentage signal changes during CVS combined with low and high attentional loads on the visual tracking task. Otherwise, same as Figure $4 b .{ }^{*} p<0.05$, ${ }^{* *} p<0.01$.

stimulation condition $\left(F_{(1,17)}=1.83, p=0.19\right.$, partial $\left.\eta^{2}=0.10\right)$ or interaction between ROI and stimulation condition $\left(F_{(1,17)}=\right.$ $0.01, p=0.91$, partial $\left.\eta^{2}=0.001\right)$. Post hoc analyses showed significant BOLD signal increases in the vestibular thalamic nuclei in both stimulation conditions compared with baseline (corresponding to central fixation with warm thermal stimulation in both ears; CVS + low load: $t_{(17)}=3.26, p=0.005, d=0.77$; CVS + high load: $\left.t_{(17)}=2.44, p=0.03, d=0.57\right)$. There was no significant difference in BOLD signal between the high and low load conditions $\left(t_{(17)}=-0.64, p=0.53, d=-0.15\right)$. Together, these results indicate that vestibular thalamic nuclei with strong connectivity with PIVC increased their activation in response to CVS regardless of the visual attentional load. These results are in strong contrast to those found for PIVC (Fig. 4b) and suggest that the attentional suppression occurred cortically rather than subcortically.

\section{Discussion}

Here, we investigated how attention acts to cross-modally suppress the processing of nonattended sensory signals. We used the suppression of the vestibular sensory system by visual attention as a model and, using fMRS, measured changes in the concentrations of chief excitatory (Glx) and inhibitory (GABA) neurotransmitters in the core cortical area of the suppressed system (PIVC) during increasing loads of visual attention. We find that the concentration of Glx in PIVC decreased during high visual attentional load compared with low visual attentional load, whereas the concentration of GABA remained stable across attentional load conditions. The decrease of Glx during high attentional load was associated with an increase in negative BOLD signal in PIVC during the same condition, such that subjects with a greater decrease of Glx tended to show a larger increase of negative BOLD from low to high visual attentional loads. This increase of negative BOLD in PIVC during high visual attentional load occurred despite simultaneously presented, excitatory vestibular cues by means of CVS. In strong contrast to 
PIVC, core vestibular nuclei of the thalamus showed an increased BOLD signal during CVS, regardless of the visual attentional load, indicating that excitatory vestibular input from the thalamus to the cortex was not suppressed. Together, our results suggest that attention acts to crossmodally suppress the processing of nonattended sensory signals by rendering the core cortical area of the suppressed system less responsive to excitatory subcortical input by downregulating Glx cortically.

Current models of visual-vestibular integration assume that visual and vestibular cues are integrated following Bayesian probability rules (MacNeilage et al., 2008; Morgan et al., 2008; Angelaki et al., 2009; Fetsch et al., 2009; Butler et al., 2010), whereby different weights are given to different sensory inputs, depending on the reliability of each input (Morgan et al., 2008). This differential weighting may then result in an attenuation or a suppression of the less weighted input (Waespe and Henn, 1978; Morgan et al., 2008). In the visual attentional tracking paradigm, we could speculate that a greater weight was assigned to the task-relevant visual input that increased with increasing tracking load. In contrast, the vestibular input was irrelevant and potentially distracting away from the tracking task, which is why a lower weight was assigned to the vestibular signals, whereby the weight decreased with increasing tracking load. This reduction of weight for vestibular signals during increasing loads of visual attentional tracking might be implemented mechanistically by a suppression of processing in PIVC. It would be interesting to investigate in future studies the weighting of visual and vestibular cues during visual attentional tracking in other areas of the vestibular cortex, in particular in regions tuned to both visual and vestibular signals such as the visual posterior Sylvian area [Chen et al., 2011; PIC as the potential human homolog (Frank et al., 2014)].

Our fMRS results show a significant decrease of Glx in PIVC during high visual attentional tracking loads compared with low visual attentional tracking loads. This agrees with previous reports showing a decrease in the concentration of $\mathrm{Glx} /$ glutamate during suppression induced by some task or transcranial stimulation (Stagg et al., 2009; Lally et al., 2014; Apšvalka et al., 2015; Martínez-Maestro et al., 2019; Boillat et al., 2020). Furthermore, our results suggest that the decrease of Glx is tightly coupled to an increase in negative BOLD in PIVC from low to high visual attentional loads, which agrees with the strong relationship between cortical glucose oxidation and glutamate-glutamine cycling (Petroff, 2002; Rothman et al., 2003). This suggests the possibility that visual attention acts to cross-modally suppress vestibular processing in PIVC by decreasing the activity of the glutamate-glutamine cycle within PIVC, which would render PIVC less responsive to excitatory thalamic input. In the glutamate-glutamine cycle, glutamate is released from the presynaptic neuron, binds to receptors of the postsynaptic neuron, and is then transported to surrounding astroglia, where it is converted to glutamine. Glutamine is transported out of the astroglia to the presynaptic neuron where it is converted back to glutamate (Rothman et al., 2003). Using fMRS, it is difficult to determine which specific components of the glutamate-glutamine cycle are suppressed by attention and whether reductions in Glx concentration result from vesicular, intracellular (synaptic), or extracellular (synaptic) neurotransmitter reduction. Given that attention operates rather quickly, on a scale of hundreds of milliseconds, it is not likely that attention alters the number of glutamate molecules present in a given voxel. Rather it might affect the concentration of available glutamate (e.g., in the synapse), perhaps by affecting the likelihood of vesicular release. How this is accomplished is an open question and requires further investigation in future studies.

Of note, we observed neither a significant change in the concentration of GABA in PIVC from the low- to the high-load attentional tracking condition nor a significant correlation between changes in GABA and BOLD between the load conditions. Although these results indicate that the suppression is not primarily induced by increased inhibitory neurotransmitter release, the following aspects need to be considered. First, previous studies have used a different experimental design for MRS and measured the concentration of GABA at rest while subjects did not perform any particular task (Duncan et al., 2014). Increased concentrations of GABA at rest were found to correlate with greater suppression in behavior or BOLD in a different task (Northoff et al., 2007; Muthukumaraswamy et al., 2009; Donahue et al., 2010; Sumner et al., 2010; Stagg et al., 2011; Hu et al., 2013), indicating that baseline GABA concentrations are associated with the magnitude of suppression of behavior or neuronal activation. Second, some previous studies found concomitant decreases of Glx or glutamate and GABA (Stagg et al., 2009; Boillat et al., 2020). This result might be accounted for by the close relationship between GABA and the excitatory glutamate-glutamine cycle (Petroff, 2002; Rothman et al., 2003). Glutamine is the precursor of glutamate, whereas glutamate is the precursor of GABA (Martin and Rimvall, 1993; Petroff, 2002; Rothman et al., 2003; Stagg, 2014). Therefore, a decrease of glutamate might also result in a decrease of GABA (see Stagg et al., 2009; Boillat et al., 2020). We could speculate that an increase in the activity of GABAergic interneurons in the current study was counteracted by the concomitant decrease of glutamate, which is why no net changes in the concentrations of GABA from low to high attentional loads were observed.

Together, the results of this study suggest that attention crossmodally acts to suppress the processing of nonattended sensory cues cortically by decreasing the amount of glutamate-glutamine within the core cortical area of the nonattended sensory system, which renders the suppressed area less responsive to excitatory subcortical input. Future research is needed to determine whether this neurochemical mechanism provides a general mechanism of active attentional cross-modality and within-modality suppression beyond the visual-vestibular model system investigated in this study.

\section{References}

Angelaki DE, Klier EM, Snyder LH (2009) A vestibular sensation: probabilistic approaches to spatial perception. Neuron 64:448-461.

Apšvalka D, Gadie A, Clemence M, Mullins PG (2015) Event-related dynamics of glutamate and BOLD effects measured using functional magnetic resonance spectroscopy (fMRS) at $3 \mathrm{~T}$ in a repetition suppression paradigm. Neuroimage 118:292-300.

Bang JW, Shibata K, Frank SM, Walsh EG, Greenlee MW, Watanabe T, Sasaki Y (2018) Consolidation and reconsolidation share behavioral and neurochemical mechanisms. Nat Hum Behav 2:507-513.

Boillat Y, Xin L, Van der Zwaag W, Gruetter R (2020) Metabolite concentration changes associated with positive and negative BOLD responses in the human visual cortex: a functional MRS study at 7 tesla. J Cereb Blood Flow Metab 40:488-500.

Bottomley PA (1984) Selective volume method for performing localized NMR spectroscopy. U.S. Patent No. 4,480,228. Schenectady, NY: General Electric.

Bottomley PA (1987) Spatial localization in NMR spectroscopy in vivo. Ann N Y Acad Sci 508:333-348. 
Brainard DH (1997) The psychophysics toolbox. Spat Vis 10:433-436.

Brandt T, Bartenstein P, Janek A, Dieterich M (1998) Reciprocal inhibitory visual-vestibular interaction. Visual motion stimulation deactivates the parieto-insular vestibular cortex. Brain 121:17491758.

Butler JS, Smith ST, Campos JL, Bülthoff HH (2010) Bayesian integration of visual and vestibular signals for heading. J Vis 10(11):23, 1-13.

Carrasco M (2011) Visual attention: the past 25 years. Vision Res 51:14841525.

Chen A, DeAngelis GC, Angelaki DE (2010) Macaque parieto-insular vestibular cortex: responses to self-motion and optic flow. J Neurosci 30:30223042.

Chen A, DeAngelis GC, Angelaki DE (2011) Convergence of vestibular and visual self-motion signals in an area of the posterior sylvian fissure. J Neurosci 31:11617-11627.

Culham JC, Cavanagh P, Kanwisher NG (2001) Attention response functions: characterizing brain areas using fMRI activation during parametric variations of attentional load. Neuron 32:737-745.

Dale AM, Fischl B, Sereno MI (1999) Cortical surface-based analysis: I. Segmentation and surface reconstruction. Neuroimage 9:179194.

De Bondt T, De Belder F, Vanhevel F, Jacquemyn Y, Parizel PM (2015) Prefrontal GABA concentration changes in women-influence of menstrual cycle phase, hormonal contraceptive use, and correlation with premenstrual symptoms. Brain Res 1597:129-138.

Deutschländer A, Bense S, Stephan T, Schwaiger M, Brandt T, Dieterich M (2002) Sensory system interactions during simultaneous vestibular and visual stimulation in PET. Hum Brain Mapp 16:92-103.

Dieterich M, Bense S, Lutz S, Drzezga A, Stephan T, Bartenstein P, Brandt T (2003) Dominance for vestibular cortical function in the non-dominant hemisphere. Cereb Cortex 13:994-1007.

Donahue MJ, Near J, Blicher JU, Jezzard P (2010) Baseline GABA concentration and fMRI response. Neuroimage 53:392-398.

Duncan NW, Wiebking C, Northoff G (2014) Associations of regional GABA and glutamate with intrinsic and extrinsic neural activity in humans - a review of multimodal imaging studies. Neurosci Biobehav Rev 47:36-52.

Fetsch CR, Turner AH, DeAngelis GC, Angelaki DE (2009) Dynamic reweighting of visual and vestibular cues during self-motion perception. J Neurosci 29:15601-15612.

Fischl B, Sereno MI, Dale AM (1999) Cortical surface-based analysis. II: inflation, flattening, and a surface-based coordinate system. Neuroimage 9:195-207.

Frank SM, Baumann O, Mattingley JB, Greenlee MW (2014) Vestibular and visual responses in human posterior insular cortex. J Neurophysiol 112:2481-2491.

Frank SM, Greenlee MW (2014) An MRI-compatible caloric stimulation device for the investigation of human vestibular cortex. J Neurosci Methods 235:208-218.

Frank SM, Greenlee MW (2018) The parieto-insular vestibular cortex in humans: more than a single area? J Neurophysiol 120:1438-1450.

Frank SM, Sun L, Forster L, Tse PU, Greenlee MW (2016a) Cross-modal attention effects in the vestibular cortex during attentive tracking of moving objects. J Neurosci 36:12720-12728.

Frank SM, Wirth AM, Greenlee MW (2016b) Visual-vestibular processing in the human Sylvian fissure. J Neurophysiol 116:263-271.

Frank SM, Pawellek M, Forster L, Langguth B, Schecklmann M, Greenlee MW (2020) Attention networks in the parietooccipital cortex modulate activity of the human vestibular cortex during attentive visual processing. J Neurosci 40:1110-1119.

Glasser MF, Coalson TS, Robinson EC, Hacker CD, Harwell J, Yacoub E, Ugurbil K, Andersson J, Beckmann CF, Jenkinson M, Smith SM, Van Essen DC (2016) A multi-modal parcellation of human cerebral cortex. Nature 536:171-178.

Hu Y, Chen X, Gu H, Yang Y (2013) Resting-state glutamate and GABA concentrations predict task-induced deactivation in the default mode network. J Neurosci 33:18566-18573.

Indovina I, Maffei V, Bosco G, Zago M, Macaluso E, Lacquaniti F (2005) Representation of visual gravitational motion in the human vestibular cortex. Science 308:416-419.
Kastner S, De Weerd P, Desimone R, Ungerleider LG (1998) Mechanisms of directed attention in the human extrastriate cortex as revealed by functional MRI. Science 282:108-111.

Kleinschmidt A, Thilo KV, Büchel C, Gresty MA, Bronstein AM, Frackowiak RS (2002) Neural correlates of visual-motion perception as object-or selfmotion. Neuroimage 16:873-882.

Kolasinski J, Logan JP, Hinson EL, Manners D, Divanbeighi Zand AP, Makin TR, Emir UE, Stagg CJ (2017) A mechanistic link from GABA to cortical architecture and perception. Curr Biol 27:1685-1691.

Krauth A, Blanc R, Poveda A, Jeanmonod D, Morel A, Székely G (2010) A mean three-dimensional atlas of the human thalamus: generation from multiple histological data. Neuroimage 49:2053-2062.

Lally N, Mullins PG, Roberts MV, Price D, Gruber T, Haenschel C (2014) Glutamatergic correlates of gamma-band oscillatory activity during cognition: a concurrent ER-MRS and EEG study. Neuroimage 85:823-833.

Lopez C, Blanke O (2011) The thalamocortical vestibular system in animals and humans. Brain Res Rev 67:119-146.

Lopez C, Blanke O, Mast FW (2012) The human vestibular cortex revealed by coordinate-based activation likelihood estimation meta-analysis. Neuroscience 212:159-179.

MacNeilage PR, Ganesan N, Angelaki DE (2008) Computational approaches to spatial orientation: from transfer functions to dynamic Bayesian inference. J Neurophysiol 100:2981-2996.

Martin DL, Rimvall K (1993) Regulation of $\gamma$-aminobutyric acid synthesis in the brain. J Neurochem 60:395-407.

Martínez-Maestro M, Labadie C, Möller HE (2019) Dynamic metabolic changes in human visual cortex in regions with positive and negative blood oxygenation level-dependent response. J Cereb Blood Flow Metab 39:2295-2307.

Mescher M, Tannus A, Johnson MN, Garwood M (1996) Solvent suppression using selective echo dephasing. J Magn Reason A 123:226-229.

Mescher M, Merkle H, Kirsch J, Garwood M, Gruetter R (1998) Simultaneous in vivo spectral editing and water suppression. NMR Biomed 11:266-272.

Morgan ML, DeAngelis GC, Angelaki DE (2008) Multisensory integration in macaque visual cortex depends on cue reliability. Neuron 59:662-673.

Muthukumaraswamy SD, Edden RA, Jones DK, Swettenham JB, Singh KD (2009) Resting GABA concentration predicts peak gamma frequency and fMRI amplitude in response to visual stimulation in humans. Proc Natl Acad Sci U S A 106:8356-8361.

Northoff G, Walter M, Schulte RF, Beck J, Dydak U, Henning A, Boeker H, Grimm S, Boesiger P (2007) GABA concentrations in the human anterior cingulate cortex predict negative BOLD responses in fMRI. Nat Neurosci 10:1515-1517.

Ogg RJ, Kingsley PB, Taylor JS (1994) WET, a T1- and B1-insensitive watersuppression method for in vivo localized 1H NMR spectroscopy. J Magn Reson B 104:1-10.

Oldfield RC (1971) The assessment and analysis of handedness: the Edinburgh inventory. Neuropsychologia 9:97-113.

Pelli DG (1997) The VideoToolbox software for visual psychophysics: transforming numbers into movies. Spat Vis 10:437-442.

Petroff OA (2002) GABA and glutamate in the human brain. Neuroscientist 8:562-573.

Provencher SW (1993) Estimation of metabolite concentrations from localized in vivo proton NMR spectra. Magn Reson Med 30:672-679.

Provencher SW (2001) Automatic quantitation of localized in vivo 1H spectra with LCModel. NMR Biomed 14:260-264.

Pylyshyn ZW, Storm RW (1988) Tracking multiple independent targets: evidence for a parallel tracking mechanism. Spat Vis 3:179-197.

Raichle ME (2015) The brain's default mode network. Annu Rev Neurosci 38:433-447.

Roberts RE, Ahmad H, Arshad Q, Patel M, Dima D, Leech R, Seemungal BM, Sharp DJ, Bronstein AM (2017) Functional neuroimaging of visuovestibular interaction. Brain Struct Funct 222:2329-2343.

Rothman DL, Behar KL, Hyder F, Shulman RG (2003) In vivo NMR studies of the glutamate neurotransmitter flux and neuroenergetics: implications for brain function. Annu Rev Physiol 65:401-427.

Roux L, Buzsáki G (2015) Tasks for inhibitory interneurons in intact brain circuits. Neuropharmacology 88:10-23. 
Saenz M, Buracas GT, Boynton GM (2002) Global effects of feature-based attention in human visual cortex. Nat Neurosci 5:631632.

Shibata K, Sasaki Y, Bang JW, Walsh EG, Machizawa MG, Tamaki M, Chang LH, Watanabe T (2017) Overlearning hyperstabilizes a skill by rapidly making neurochemical processing inhibitory-dominant. Nat Neurosci 20:470-475.

Stagg CJ (2014) Magnetic resonance spectroscopy as a tool to study the role of GABA in motor-cortical plasticity. Neuroimage 86:19-27.

Stagg CJ, Best JG, Stephenson MC, O’Shea J, Wylezinska M, Kincses ZT, Morris PG, Matthews PM, Johansen-Berg H (2009) Polarity-sensitive modulation of cortical neurotransmitters by transcranial stimulation. J Neurosci 29:5202-5206.

Stagg CJ, Bachtiar V, Johansen-Berg H (2011) The role of GABA in human motor learning. Curr Biol 21:480-484.

Stanley JA, Raz N (2018) Functional magnetic resonance spectroscopy: the "new" MRS for cognitive neuroscience and psychiatry research. Front Psychiatry 9:76.
Sumner P, Edden RA, Bompas A, Evans CJ, Singh KD (2010) More GABA, less distraction: a neurochemical predictor of motor decision speed. Nat Neurosci 13:825-827.

Suzuki M, Kitano H, Ito R, Kitanishi T, Yazawa Y, Ogawa T, Shiino A, Kitajima K (2001) Cortical and subcortical vestibular response to caloric stimulation detected by functional magnetic resonance imaging. Brain Res Cogn Brain Res 12:441-449.

Tootell RB, Hadjikhani N, Hall EK, Marrett S, Vanduffel W, Vaughan JT, Dale AM (1998) The retinotopy of visual spatial attention. Neuron 21: 1409-1422.

Waespe W, Henn V (1978) Conflicting visual-vestibular stimulation and vestibular nucleus activity in alert monkeys. Exp Brain Res 33:203-211.

Wirth AM, Frank SM, Greenlee MW, Beer AL (2018) White matter connectivity of the visual-vestibular cortex examined by diffusion-weighted imaging. Brain Connect 8:235-244. 